\title{
Differentiation between non-hypervascular pancreatic neuroendocrine tumour and pancreatic ductal adenocarcinoma on dynamic computed tomography and non-enhanced magnetic resonance imaging
}

\author{
Kazuyoshi Ohki ${ }^{A, D, E, F}$, Takao Igarashi ${ }^{A, C D}$, Hirokazu Ashida ${ }^{D}$, Megumi Shiraishi ${ }^{B}$, Yosuke Nozawa ${ }^{F}$, Hiroya Ojiri ${ }^{F}$ \\ The Jikei University School of Medicine, Japan
}

\section{Abstract}

Purpose: To determine the differentiating features between non-hypervascular pancreatic neuroendocrine tumour (PNET) and pancreatic ductal adenocarcinoma (PDAC) on dynamic computed tomography (CT) and non-enhanced magnetic resonance imaging (MRI).

Material and methods: We enrolled 102 patients with non-hypervascular PNET ( $n=15)$ or PDAC $(n=87)$, who had undergone dynamic CT and non-enhanced MRI. One radiologist evaluated all images, and the results were subjected to univariate and multivariate analyses. To investigate reproducibility, a second radiologist re-evaluated features that were significantly different between PNET and PDAC on multivariate analysis.

Results: Tumour margin (well-defined or ill-defined) and enhancement ratio of tumour (ERT) showed significant differences in univariate and multivariate analyses. Multivariate analysis revealed a predominance of well-defined tumour margins in non-hypervascular PNET, with an odds ratio of 168.86 (95\% confidence interval [CI]: 10.62-2685.29; $p<0.001)$. Furthermore, ERT was significantly lower in non-hypervascular PNET than in PDAC, with an odds ratio of 85.80 (95\% CI: $2.57-2860.95 ; p=0.01$ ). Sensitivity, specificity, and accuracy were $86.7 \%, 96.6 \%$, and $95.1 \%$, respectively, when the tumour margin was used as the criteria. The values for ERT were $66.7 \%, 98.9 \%$, and $94.1 \%$, respectively. In reproducibility tests, both tumour margin and ERT showed substantial agreement (margin of tumour, $\kappa=0.6356$; ERT, intraclass correlation coefficients (ICC) $=0.6155)$.

Conclusions: Non-hypervascular PNET showed well-defined margins and lower ERT compared to PDAC, with significant differences. Our results showed that non-hypervascular PNET can be differentiated from PDAC via dynamic CT and non-enhanced MRI.

Key words: magnetic resonance imaging, tomography, X-ray computed, pancreatic neoplasms; carcinoma, pancreatic ductal, neuroendocrine tumours, adenoma, islet cell.

\section{Introduction}

Pancreatic ductal adenocarcinoma (PDAC) is one of the most aggressive cancers. It has a poor prognosis, and the five-year survival rate is less than $4 \%$ [1-3]. This high mortality rate is due to the cancer's biological aggressiveness and advanced state at the time of diagnosis [4]. In recent years, the incidence of PDAC has been increasing, and it is projected to be the second most common cause of cancer-related death in the United States in 2020 [5].

Meanwhile, pancreatic neuroendocrine tumour (PNET) is a rare pancreatic tumour originating from pluripotent stem cells in the ductal epithelium [2,6]. PNET is pathologically classified into G1, G2, and G3 according to Ki-67 index. G1 and G2 indicate well-differentiated PNET, and G3 indicates a poorly differentiated neuroendocrine carci-

Correspondence address:

Kazuyoshi Ohki, Department of Radiology, The Jikei University School of Medicine, 3-25-8, Nishi-Shimbashi, Minato-ku, Tokyo 105-8461, Japan,

phone: +81-3-3433-1111, fax: +81-3-3431-1775, e-mail: ms99-oki@jikei.ac

Authors' contribution:

A Study design · B Data collection · C Statistical analysis · D Data interpretation · E Manuscript preparation · F Literature search · G Funds collection 
noma [7]. The five-year survival rate ranges from $60-100 \%$ for well-differentiated PNET to $29 \%$ for poorly differentiated PNET [8].

Surgery is the only curative treatment for patients with PNET or PDAC. Patients with PDAC need a more radical surgery that includes lymphadenectomy $[4,9,10]$, while those with PNET, which is non-invasive and smaller in size $(<20 \mathrm{~mm})$, may only require a limited resection without lymphadenectomy, such as tumour enucleation, central pancreatectomy, or laparoscopic surgery [10]. Therefore, it is important to differentiate between PNET and PDAC preoperatively to estimate the prognosis and plan a surgical strategy.

PNET usually shows arterial enhancement with progressively decreased enhancement [2,3,6,11]. Although clinicians can easily diagnose PNET on preoperative imaging because, other than PNET, the number of hypervascular pancreatic tumours is very small [11], up to $48.6 \%$ of PNET does not show arterial enhancement, as is common in most PDAC $[9,12,13]$. Therefore, non-hypervascular PNET can be a differential diagnosis during assessment for PDAC via imaging.

Only a few studies have focused on the differences between non-hypervascular PNET and PDAC found on diagnostic images. The purpose of this study was to evaluate the findings on dynamic computed tomography (CT) and non-enhanced magnetic resonance imaging (MRI) for differentiation between non-hypervascular PNET and PDAC.

\section{Material and methods}

\section{Patient selection}

This retrospective cohort study was approved by the review board of our institution, and the need for informed consent was waived. The records of the patients with pathologically proven PNET or PDAC were reviewed when clinical information was available. All patients underwent surgery, including pancreatoduodenectomy, distal pancreatectomy, central pancreatectomy, and tumour enucleation, between April 2011 and June 2017. According to the European Society for Medical Oncology (ESMO) Clinical Practice Guidelines, dynamic CT plays a central role in the evaluation of pancreatic tumour, especially when pancreatic cancer is suspected, and should be the first choice of imaging investigation [14]. Furthermore, MRI could be useful as a supplementary imaging modality to evaluate vessel involvement and biliary anatomy, detect liver metastasis not detected by CT, and differentiate cystic lesions [14]. Based on the literature, we evaluated pancreatic tumours with dynamic CT and non-enhanced MRI. The inclusion criteria were as follows: (a) patients who underwent dynamic CT and non-enhanced MRI according to our institutional routine protocol within six months before surgery and (b) patients who had a detectable tumour, and the region of interest (ROI) could be set via CT and MRI. Meanwhile, the exclusion criteria were patients who had tumours with visually higher enhancement than pancreatic parenchyma on arterial phase.

\section{CT scanning protocol}

All CT images were obtained using either a 64-channel scanner (SOMATOM Perspective, Siemens, $n=34$ ) or a 128-channel scanner (SOMATOM Definition Flash, $n=32$ or SOMATOM Definition AS+, Siemens, $n=36$ ). The scanning protocol consisted of non-enhanced and biphasic contrast-enhanced scans. The non-enhanced phase was obtained through the upper abdomen, including the entire liver and pancreas. Then, a bolus of $600 \mathrm{mgI} / \mathrm{kg}$ of iodine contrast medium was administered using an automatic power injector at a rate of $2.0-3.0 \mathrm{ml} / \mathrm{s}$ for 33 seconds. The contrast media used included iohexol (Omnipaque 350 Injection, Daiichi Sankyo, $n=34$ or IOVERIN 350, Teva Takeda Pharma, $n=25$ ), iopamidol (Iopamiron 370 Inj., Bayer Yakuhin, $n=20$ or Oypalomin 370 injection, Fuji Pharma, $n=18$ ), or iomeprol (Iomeron 350 , Eisai, $n=5$ ). Arterial phase was obtained 10 seconds after reaching 80 Hounsfield units with the ROI placed on the aorta at the level of the celiac artery, while portal venous phase was obtained 90 seconds after triggering. The arterial and portal venous phases were obtained through the upper abdomen and through the whole abdomen, respectively.

\section{MRI scanning protocol}

All MRI images were acquired using a 1.5-T whole-body MRI system with a six-channel phased array as the receiver coil (MAGNETOM Avanto, Siemens, $n=43$ or MAGNETOM Symphony, A Tim System, Siemens, $n=59$ ). The following objects were analysed in the routine abdominal MRI protocol: transverse T1-weighted image (T1WI) using a fat-saturated 2D or 3D gradient echo (2D fast low-angle shot or 3D volumetric interpolated breath-hold sequence; transverse T2-weighted image (T2WI) with fast spin-echo; and transverse diffusion-weighted image (DWI) performed as a single-shot echo-planar imaging pulse sequence with $b$-values of 50 and $800 \mathrm{sec} / \mathrm{mm}^{2}$ using respiratory triggering. The apparent diffusion coefficient (ADC) was calculated with $b$ values of 50 and $800 \mathrm{sec} / \mathrm{mm}^{2}$. The MRI pulse sequence parameters are summarised in Table 1.

\section{Imaging analysis}

An abdominal radiologist with 11 years of experience, who was blinded to pathological diagnosis and clinical information, retrospectively reviewed anonymised dynamic CT and non-enhanced MRI images on a Picture Archiving and Communication Systems workstation monitor. 
Table 1. Pulse sequence parameters for 1.5-T MR imaging

\begin{tabular}{|l|c|c|c|c|}
\hline Factor & 2D FLASH & 3D VIBE & T2WI & DWI \\
\hline TR (ms) & $102-142$ & $4.94-5.20$ & 3000 & $1200-1500$ \\
\hline TE $(\mathrm{ms})$ & $4.76-5.04$ & $1.86-1.93$ & $90-91$ & $67-86$ \\
\hline FA (degrees) & 70 & 15.0 & 150 & $50 / 800$ \\
\hline$b$ values & & & & $77 \times 128$ \\
\hline Matrix & $187 \times 256$ & $199 \times 384$ & $208 \times 320-256 \times 320$ & $262 \times 350-277 \times 370$ \\
\hline FOV & $308 \times 380-308 \times 384$ & $300 \times 400$ & $308 \times 380$ & 7.0 \\
\hline ST (mm) & 7.0 & 3.0 & 601 & $1346-2298$ \\
\hline BW (Hz/pixel) & 300 & 260 & 30 & $2: 09-2: 26$ \\
\hline AT (s) & 38 & $19-20$ & 31 & \\
\hline ETL & & & & \\
\hline
\end{tabular}

FLASH - fast low-angle shot, VIBE - volumetric interpolated breath-hold examination (T1-weighted gradient-echo sequences, which are referred to as "FLASH" and "VIBE" on Siemens Healthcare MRI systems), T2WI - T2-weighted imaging, DWI - diffusion-weighted imaging, TR - repetition time, TE - echo time, FA - flip angle, FOV - field of view, ST - slice thickness, BW - bandwidth, AT - acquisition time, ETL - echo train length, MR - magnetic resonance

For qualitative analysis, the following imaging parameters were evaluated: (a) tumour margin (well-defined or ill-defined), (b) cystic change or necrosis (present or absent), (c) calcification (present or absent), (d) upstream pancreatitis (present or absent), and (e) dilated main pancreatic duct (present or absent). Upstream pancreatitis was defined as pancreatic parenchyma showing high intensity on DWI and low intensity on fat-saturated T1WI. The dilated main pancreatic duct was defined as positive when its diameter was more than $3 \mathrm{~mm}$. Pancreatic parenchyma was defined as a presumed non-pathological pancreatic region in which focal abnormalities such as pancreatitis and/or cystic lesions were not included.

For quantitative analysis, the following parameters were evaluated: (a) tumour size, (b) CT attenuation and MRI signal intensity of tumour and pancreatic parenchyma, (c) ADC values of tumour, (d) CT attenuation and MRI signal intensity ratios of tumour to pancreatic parenchyma, and (e) enhancement ratio of tumour (ERT). ROI was set to the largest solid portion of the tumour, avoiding the cystic component on CT images of non-enhanced, arterial phase, and portal venous phase, and on MRI images of fat-saturated T1WI, T2WI, DWI, and ADC map. We measured the density and signal intensity three times by ROI, and the average value was calculated. The CT attenuation and MRI signal intensity ratios of tumour to pancreatic parenchyma were calculated using the following formula: mean CT attenuation or MRI signal intensity of tumour/mean CT attenuation or MRI signal intensity of pancreatic parenchyma. The ERT during arterial and portal venous phases were calculated as follows: (Tp-Ta)/ ( Ta-Tn), where $\mathrm{Tn}, \mathrm{Ta}$, and $\mathrm{Tp}$ were the attenuation of the tumour in Hounsfield units, measured during non-enhanced, arterial, and portal venous phase, respectively.

To assess the reproducibility of significant variables, a second radiologist with three years of experience in abdominal imaging evaluated variables that showed significant differences on multivariate analyses, while being blinded to the pathological and clinical information.

\section{Statistical analysis}

Fisher's exact test was used for qualitative variables, and the Mann-Whitney $U$ test was used for quantitative analysis. Multivariate logistic regression analysis was performed using variables estimated to be related to outcomes based on knowledge and clinical judgment from previous reports $[6,10,11,13]$.

The diagnostic performance of each quantitative variable was estimated via receiver operating characteristic (ROC) analysis. The optimal thresholds for differentiating between the PNET group and the PDAC group were chosen at the highest possible sensitivity and specificity on the ROC curves. Variables set with optimal thresholds were fit to the multivariate logistic regression analysis. Statistical analysis was executed using Ekuseru-Toukei 2015 (SSRI, Tokyo, Japan) and R (The R Project for Statistical Computing, version 3.3.0). For all tests, a $p$-value of less than 0.05 was considered statistically significant. Inter-observer agreement of findings was evaluated by calculating $\kappa$ values for dichotomous variables or intraclass correlation coefficients (ICC) for continuous variables. The $\kappa$ values and ICC were classified as follows: 1.0 was considered to indicate perfect agreement; 0.81-0.99, almost perfect agreement; 0.61-0.80, substantial agreement; 0.41 0.60 , moderate agreement; $0.21-0.40$, fair agreement; and $\leq 0.20$, slight agreement.

\section{Results}

A total of 102 patients (15 patients with non-hypervascular PNET and 87 patients with PDAC) met the inclusion 


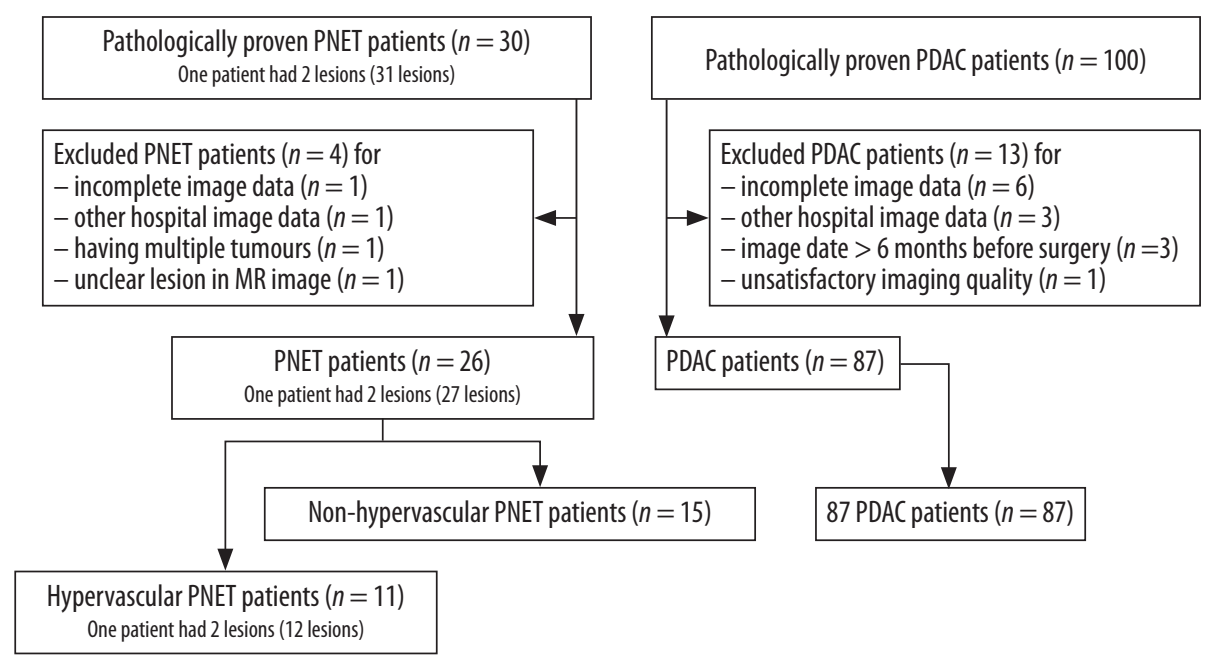

PNET - pancreatic neuroendocrine tumour, PDAC - pancreatic ductal adenocarcinoma, MR - magnetic resonance

Figure 1. Flow diagram summarising the patient sampling process

Table 2. Results of patient and tumour characteristics

\begin{tabular}{|c|c|c|c|}
\hline Variable & PNET $(n=15)$ & $\operatorname{PDAC}(n=87)$ & $p$-value \\
\hline Age (y) & $51(42-63.5)$ & $69(63-76)$ & $<0.001$ \\
\hline \multicolumn{4}{|l|}{ Gender } \\
\hline Male & $8(53)$ & $50(57)$ & \multirow[t]{2}{*}{0.78} \\
\hline Female & $7(47)$ & $37(43)$ & \\
\hline \multicolumn{4}{|l|}{ Tumour location } \\
\hline Head & $3(20)$ & $50(57)$ & \multirow[t]{3}{*}{0.01} \\
\hline Body & $9(60)$ & $21(24)$ & \\
\hline Tail & $3(20)$ & $16(18)$ & \\
\hline Tumour size (mm) & $16(13-25.5)$ & $25(18.5-33)$ & 0.04 \\
\hline
\end{tabular}

\section{Numbers in parentheses are percentages.}

Age and size are expressed as median and interquartile range (25-75 percentile).

PNET - pancreatic neuroendocrine tumour, PDAC - pancreatic ductal adenocarcinoma

criteria (Figure 1). All included patients each had one lesion. No patients with hypervascular PDAC were identified. The cohort consisted of 58 men and 44 women, with an average age of 66 years (range: $31-84$ years). The surgeries performed in this study were pancreatoduodenectomy, distal pancreatectomy, central pancreatectomy, and tumour enucleation; the breakdown was as follows: $3(20 \%), 7(47 \%), 3(20 \%)$, and 2 (13\%) patients with PNET and 51 (59\%), 36 (41\%), 0, and 0 patients with PDAC underwent such surgeries, respectively. Based on the 2010 World Health Organisation classification system, of 15 PNETs, five tumours (33.3\%) were classified as G1, eight (55.3\%) were classified as G2, and two (13.3\%) were classified as G3.

The results of patient and tumour characteristic analyses are summarised in Table 2. The mean ROI of the tumours was $121.5 \pm 88.5 \mathrm{~mm}^{2}$. Age and tumour size showed significant differences. PNET patients were younger $(p<0.001)$, and their tumour sizes were smaller than those of PDAC patients $(p=0.04)$.

The results of quantitative and qualitative assessments are presented in Table 3. The well-defined margin was significantly different between non-hypervascular PNET and PDAC $(p<0.001)$. Only two PNETs $(13.3 \%)$ showed ill-defined margin. These two tumours were poorly differentiated neuroendocrine carcinomas classified as histopathological G3. The remaining 13 tumours were G1 or G2. The absence of both upstream pancreatitis and dilated main pancreatic duct was significant between non-hypervascular PNET and PDAC $(p<0.001)$ (Figures 2-4). On univariate analysis, CT attenuation ratio of tumour to pancreatic parenchyma on arterial phase $(p<0.001)$ and ERT $(p<0.001)$ showed a significant difference, but $\mathrm{CT}$ attenuation ratio of tumour to pancreatic parenchyma on portal venous phase did not $(p=0.05)$. MRI signal intensity ratio of tumour to pancreatic parenchyma on all sequences and ADC also did not show a significant difference on univariate analysis.

The results of multivariate analyses are summarised in Table 4. There was a significant difference in tumour margin between PNET and PDAC (odds ratio: 168.86; 95\% confidence interval [CI]: 10.62-2685.29; $p<0.001$ ). ERT was also significantly different between groups (odds ratio: 85.80 ; 95\% CI: 2.57-2860.95; $p=0.01$ ). Although not significant, the quantitative assessment of CT attenuation ratio of tumour to pancreatic parenchyma on arterial phase showed relatively good discriminative performance on multivariate analyses (odds ratio: 5.31 ; 95\% CI: 0.40$71.17 ; p=0.21$ ). The sensitivity, specificity, and accuracy of tumour margin was $86.7 \%, 96.6 \%$, and $95.1 \%$, respectively, while it was $66.7 \%, 98.9 \%$, and $94.1 \%$, respectively, for ERT.

The results of reproducibility tests of significant variables showed that the $\kappa$ value for tumour margin was 0.64 , 
Table 3. Results of quantitative and qualitative assessment

\begin{tabular}{|c|c|c|c|}
\hline Variable & PNET $(n=15)$ & $\operatorname{PDAC}(n=87)$ & $p$-value \\
\hline \multicolumn{4}{|l|}{ Tumour margin } \\
\hline Well-defined & $13(87)$ & $3(3)$ & \multirow[t]{2}{*}{$<0.001$} \\
\hline III-defined & $2(13)$ & $84(97)$ & \\
\hline \multicolumn{4}{|l|}{ Cystic change or necrosis } \\
\hline Absent & $12(80)$ & $62(71)$ & \multirow[t]{2}{*}{0.75} \\
\hline Present & $3(20)$ & $25(29)$ & \\
\hline \multicolumn{4}{|l|}{ Calcification } \\
\hline Absent & $13(87)$ & $82(94)$ & \multirow[t]{2}{*}{0.27} \\
\hline Present & $2(13)$ & $5(6)$ & \\
\hline \multicolumn{4}{|l|}{ Upstream pancreatitis } \\
\hline Absent & $14(93)$ & $41(47)$ & \multirow[t]{2}{*}{$<0.001$} \\
\hline Present & $1(7)$ & $46(53)$ & \\
\hline \multicolumn{4}{|l|}{ Dilated main pancreatic duct } \\
\hline Absent & $13(87)$ & $25(29)$ & \multirow[t]{2}{*}{$<0.001$} \\
\hline Present & $2(13)$ & $62(71)$ & \\
\hline Non-enhanced phase ${ }^{a}$ & $0.881(0.791-0.930)$ & $0.897(0.755-1.000)$ & 0.59 \\
\hline Arterial phase $\mathrm{e}^{\mathrm{a}}$ & $0.866(0.650-1.066)$ & $0.585(0.508-0.645)$ & $<0.001$ \\
\hline Portal venous phase ${ }^{a}$ & $1.013(0.898-1.233)$ & $0.921(0.791-1.062)$ & 0.05 \\
\hline Enhancement ratio of tumour (ERT) ${ }^{b}$ & $0.000(-0.152-0.701)$ & $1.000(0.681-1.433)$ & $<0.001$ \\
\hline Fat-saturated T1-weighted image & $0.649(0.540-0.777)$ & $0.665(0.596-0.752)$ & 0.80 \\
\hline T2-weighted image ${ }^{\mathrm{a}}$ & $1.279(1.049-1.493)$ & $1.190(1.052-1.387)$ & 0.62 \\
\hline Diffusion-weighted image ${ }^{\mathrm{a}}$ & 1.577 (1.409-1.941) & $1.500(1.220-1.860)$ & 0.44 \\
\hline $\mathrm{ADC}\left(\times 10^{-3} \mathrm{~mm}^{2} / \mathrm{sec}\right)$ & $1.214(1.010-1.437)$ & $1.283(1.159-1.412)$ & 0.40 \\
\hline
\end{tabular}

${ }^{2} \mathrm{C} T$ attenuation or MR signal intensity ratio of tumour to pancreatic parenchyma

bEnhancement ratio of tumour $=(\mathrm{Tp}-\mathrm{Ta}) /(\mathrm{Ta}-\mathrm{Tn})$

Numbers in parentheses are percentages.

Tn, Ta, and Tp are the attenuation of tumour (in Hounsfield Units) during non-enhanced, arterial, and portal venous phase measured, respectively.

Continuous variables are expressed as median and interquartile range (25-75 percentile).

PNET - pancreatic neuroendocrine tumour, PDAC - pancreatic ductal adenocarcinoma, ADC - apparent diffusion coefficient, CT - computed tomography, MR - magnetic resonance

and the ICC for ERT was 0.62. Both results were in substantial agreement.

\section{Discussion}

We evaluated the findings of dynamic CT and non-enhanced MRI that contributed to differentiation between non-hypervascular PNET and PDAC. We also conducted blinded observer tests to assess the reproducibility of such imaging findings.

Our results showed that there was a significant difference in well-defined margins between non-hypervascular PNET and PDAC $(p<0.001)$. These results were similar to previous studies that reported the same morphological tendency of hypervascular PNET $[6,10,11]$. The presence of well-defined margins was also a useful feature for dif- ferentiating between PDAC and PNET, even when exclusive to the non-hypervascular type, when dynamic contrast enhancement was inconclusive. Additionally, in our study, tumours with ill-defined margins were either PDAC or non-hypervascular G3 PNET. Although G3 PNET was identified in two cases, these results could also indicate that radical surgery should be considered if a patient presents with a pancreatic tumour with an ill-defined margin on preoperative imaging, because it increases the possibility of non-hypervascular G3 PNET or PDAC.

Our results show that ERT of non-hypervascular PNET is significantly lower than that of PDAC. ERT is considered to be affected by the degree of tumour fibrosis because abundant fibrosis reduces blood inflow to the tumour [15]. Histologically, PNET shows a lower degree of fibrosis, while PDAC shows abundant internal 

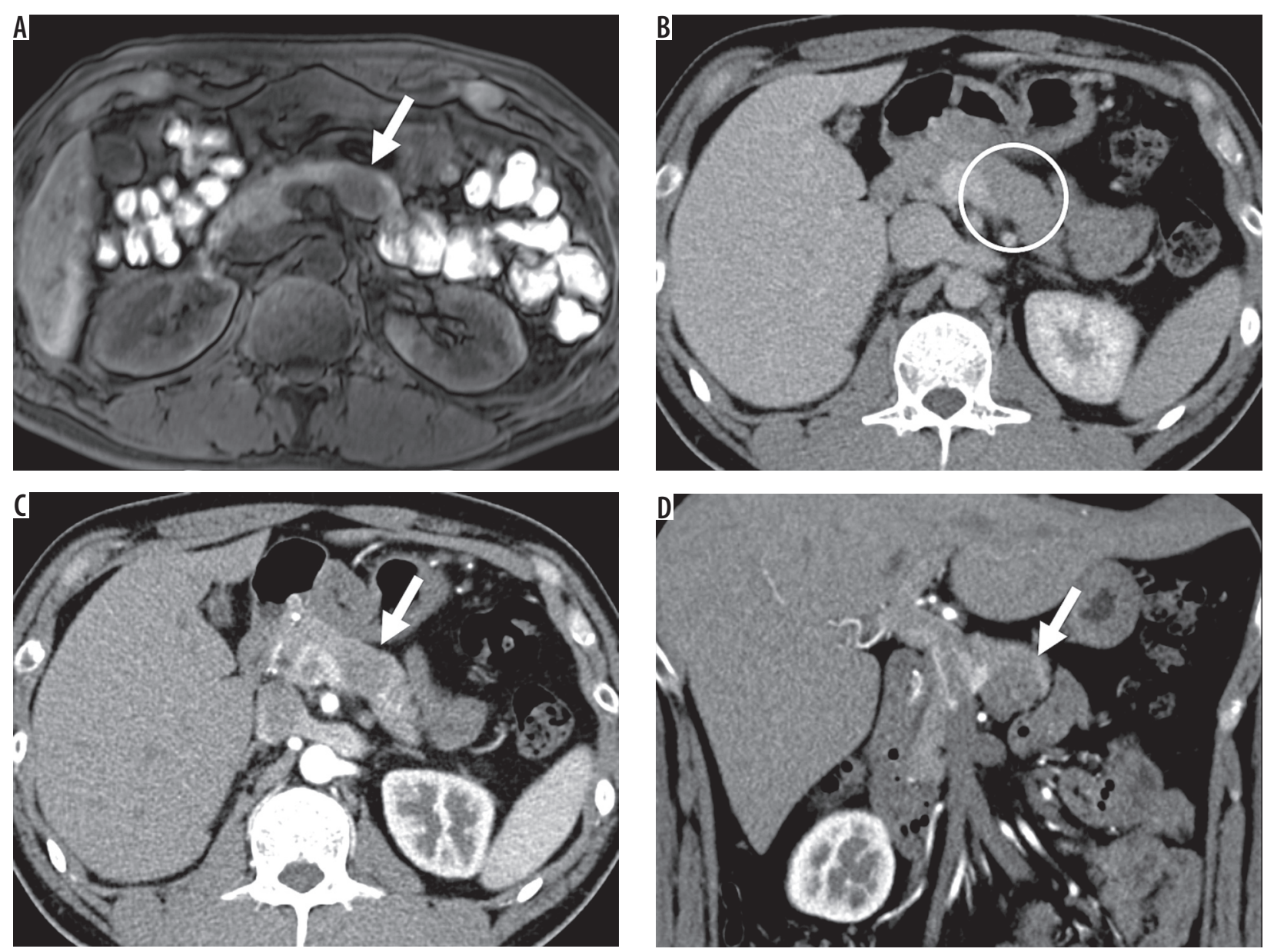

Figure 2. Imaging results from a 36-year-old man with non-hypervascular pancreatic neuroendocrine tumour (PNET) in the body of the pancreas. A) Fat-saturated T1-weighted image shows well-defined hypointense lesion (arrow). B) The enhanced portal venous phase computed tomography image shows iso-enhancement of the lesion relative to the adjacent parenchyma; therefore, the lesion is difficult to detect (circle) (C) and (D). The enhanced arterial phase computed tomography image shows that the lesion is hypoenhanced relative to the adjacent parenchyma and well-defined (arrow)

fibrosis $[12,16]$. As such, it is assumed that non-hypervascular PNET shows lower ERT than PDAC. ERT is not affected by attenuation changes of the surrounding pancreatic parenchyma caused by tumour-induced pancreatitis, and it could be calculated using only the attenuation of the tumour. Therefore, the use of ERT is valuable in differentiating between PNET and PDAC. Jeon et al. [13] reported that hyper- or iso-enhancement in the portal venous phase are useful for differentiating between non-hypervascular PNET and PDAC. They also reported a line chart analysis of temporal contrast-to-noise ratio (CNR), which is the enhancement ratio of the tumour adjusted by the paraspinal muscle for standardisation [13]. The line chart analysis of temporal CNR of PDAC showed a higher rate of contrast enhancement over time than that of non-hypervascular PNET. This was similar to our results that showed a lower ERT on non-hypervascular PNET and a higher ERT on PDAC. Our results on ERT also supported their results in the line chart analysis of temporal CNR as a dynamic curve. However, our results cannot be directly compared to their results because our
CT acquisition times for arterial and portal venous phases were different from those used in their protocol, and we used ERT as a variable, rather than a dynamic curve.

The CT attenuation ratio of tumour to pancreatic parenchyma on arterial phase showed a relatively good discriminative performance on multivariate analyses, although not significant $(p=0.21)$. This could indicate that non-hypervascular PNET shows a substantially higher enhancement degree compared with PDAC in arterial phase on quantitative analysis, even though both nonhypervascular PNET and PDAC showed a similar internal enhancement in arterial phase on visual assessment. This may reflect that the inherent histopathological property of the rich capillary network of PNET is associated with vascularity in the arterial phase [6].

A previous study by d'Assignies et al. showed that the blood flow of PNET is related to its grade, and that blood flow was significantly higher in the group of benign tumours [17]. In our study, $86.7 \%$ (13/15) of tumours were low-grade tumours classified as G1 or G2. Although excluded from this study, all hypervascular PNET were 

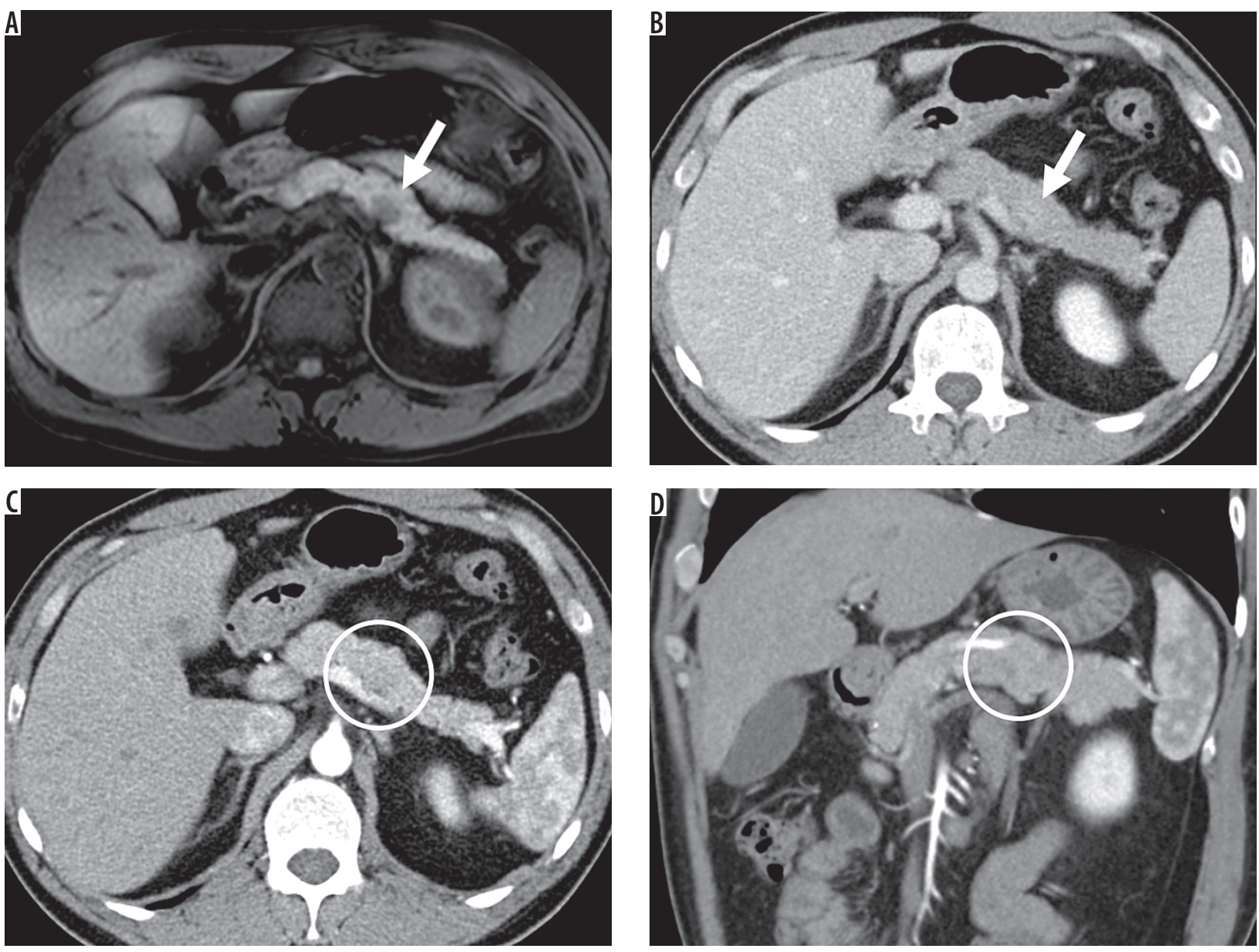

Figure 3. Imaging results from a 41-year-old man with non-hypervascular pancreatic neuroendocrine tumour (PNET) in the body of the pancreas. A) Fatsaturated T1-weighted image shows well-defined hypointense lesion (arrow). B) The enhanced portal venous phase computed tomography image shows weak enhancement of the adjacent parenchyma (arrow) $(C)$ and $(\mathbf{D})$. The enhanced arterial phase computed tomography image shows iso-enhancement of the lesion relative to the adjacent parenchyma; therefore, the lesion is difficult to detect (circle)

low-grade tumours; of 12 tumours, 11, 1, and 0 were G1, G2, and G3, respectively. Our results agreed with those of d'Assignies et al., as the proportion of G2 and G3 tumours increased in non-hypervascular PNET compared to the hypervascular PNET. However, $86.7 \%$ of tumours among non-hypervascular PNET were low-grade tumours. Moreover, our results were similar to findings of a study by Jeon et al., in which low-grade tumours accounted for $89 \%$ of non-hypervascular PNET [13]. Although non-hypervascular PNET is more likely than hypervascular PNET to include high grade tumours, the majority of non-hypervascular PNET are low-grade tumours that require less extensive surgery; thus, it is clinically important to differentiate non-hypervascular PNET from PDAC.

Little is known about the added value of non-enhanced MRI for differentiation between PNET and PDAC. In our study, the signal intensity of MRI including DWI and ADC showed no significant difference between PNET and PDAC. Such a result was similar to previous reports $[11,18]$. Concerning PNET, some studies showed that DWI and ADC have predictive value for tumour grading.
This is particularly useful to differentiate between G1-2 and G3 tumours [19-21].

The limitations of our study are its single-site retrospective design and the higher number of PDAC patients than non-hypervascular PNET. Non-hypervascular PNET is the less frequent type of PNET, which is generally a rare tumour, and only 15 cases of non-hypervascular PNET were seen in our study. Future studies with larger sample sizes are required to provide additional accuracy to our study. Furthermore, our study included all grades of PNET. Some previous studies reported that DWI is useful for the differentiation between G1-2 and G3 tumours [19-21]. Thus, if we subdivide the cases into different grades of PNET (G1-3) and PDAC prior to evaluation, there may be some imaging features that can differentiate these tumours. Additionally, our arterial phase protocol was conducted earlier than recommended in the European Society for Medical Oncology (ESMO) Clinical Practice Guidelines $[4,14]$. In our CT imaging protocol, the arterial phase was taken 10 seconds after reaching 80 Hounsfield units with ROI placed on the aorta. This 

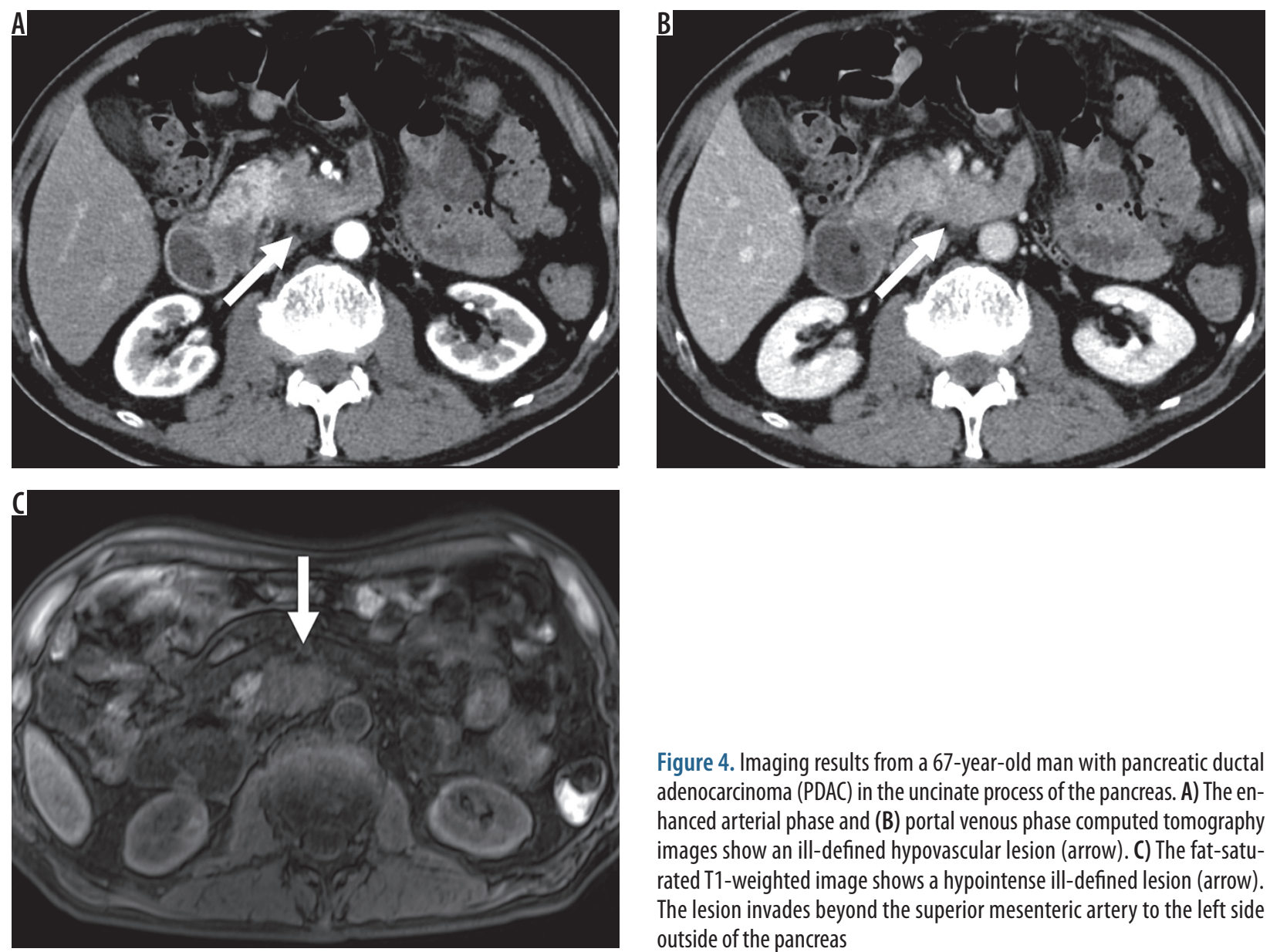

Figure 4. Imaging results from a 67-year-old man with pancreatic ductal adenocarcinoma (PDAC) in the uncinate process of the pancreas. A) The enhanced arterial phase and (B) portal venous phase computed tomography images show an ill-defined hypovascular lesion (arrow). C) The fat-saturated T1-weighted image shows a hypointense ill-defined lesion (arrow). The lesion invades beyond the superior mesenteric artery to the left side outside of the pancreas

Table 4. Results of multivariate analysis

\begin{tabular}{|l|c|c|c|c|}
\hline Variable & Odds ratio & \multicolumn{2}{|c|}{$95 \%$ Cl } & $p$-value \\
\hline Tumour margin (well-defined vs. ill-defined) & 168.86 & 10.62 & 2685.29 & $<0.001$ \\
\hline CT attenuation ratio of tumour to pancreatic parenchyma on arterial phase ( $\geq 0.64$ vs. $<0.64)$ & 5.31 & 0.40 & 71.17 & 0.21 \\
\hline Enhancement ratio of tumour (ERT) ${ }^{\mathrm{a}}(\leq 0.25$ vs. $>0.25)$ & 85.80 & 2.57 & 2860.95 & 0.01 \\
\hline
\end{tabular}

aEnhancement ratio of tumour $=(\mathrm{T} p-\mathrm{Ta}) /(\mathrm{Ta}-\mathrm{Tn})$

$\mathrm{Tn}, \mathrm{Ta}$, and $\mathrm{Tp}$ are the attenuation of tumour (in Hounsfield Units) during non-enhanced, arterial, and portal venous phase measured, respectively.

Cl - confidence interval, ERT - enhancement ratio of tumour, CT - computed tomography

was approximately 30 seconds after administration of the contrast agent, which is earlier than the 40 seconds recommended in the ESMO Clinical Practice Guidelines. The pancreatic arterial phase should also be acquired to better compare CT imaging findings to previous reports.

In conclusion, a well-defined margin and a lower ERT of non-hypervascular PNET contributed to differentiation between non-hypervascular PNET and PDAC. Consequently, by interpreting the images correctly, unnecessary extensive surgery can be avoided.

\section{Conflict of interest}

The authors report no conflict of interest.

References

1. Siegel R, Ward E, Brawley O, et al. Cancer statistics, 2011: The impact of eliminating socioeconomic and racial disparities on premature cancer deaths. CA Cancer J Clin 2011; 61: 212-236.

2. Ansari NA, Ramalho M, Semelka RC, et al. Role of magnetic resonance imaging in the detection and characterization of solid pancreatic nodules: an update. World J Radiol 2015; 7: 361-374.
3. Konstantinidis IT, Warshaw AL, Allen JN, et al. Pancreatic ductal adenocarcinoma: is there a survival difference for $\mathrm{R} 1$ resections versus locally advanced unresectable tumors? What is a "true" R0 resection? Ann Surg 2013; 257: 731-736.

4. Al-Hawary MM, Francis IR, Chari ST, et al. Pancreatic ductal adenocarcinoma radiology reporting template: consensus statement of 
the Society of Abdominal Radiology and the American Pancreatic Association. Radiology 2014; 270: 248-260.

5. Matrisian LM, Aizenberg R, Rosenzweig A. The alarming rise of pancreatic cancer deaths in the United States: why we need to stem the tide today. 2012. Available from: http://www.pancan.org/section_research/reports/pdf/incidence_report_2012.pdf (Accessed: 17 May 2013).

6. Lewis RB, Lattin GE Jr, Paal E. Pancreatic endocrine tumors: radiologic-clinicopathologic correlation. Radiographics 2010; 30: 1445-1464.

7. Klimstra DS, Modlin IR, Coppola D, et al. The pathologic classification of neuroendocrine tumors: a review of nomenclature, grading, and staging systems. Pancreas 2010; 39: 707-712.

8. Plöckinger U, Rindi G; European Neuroendocrine Tumour Society. Guidelines for the diagnosis and treatment of neuroendocrine gastrointestinal tumours: a consensus statement on behalf of the European Neuroendocrine Tumour Society (ENETS). Neuroendocrinology 2004; 80: 394-424.

9. Manfredi R, Bonatti M, Mantovani W, et al. Non-hyperfunctioning neuroendocrine tumors of the pancreas: MR imaging appearance and correlation with their biological behaviour. Eur Radiol 2013; 23: 3029-3039.

10. Sahani DV, Bonaffini PA, Fernández-Del Castillo C, et al. Gastroenteropancreatic neuroenodcrine tumors: role of imaging in diagnosis and management. Radiology 2013; 266: 38-61.

11. Jang KM, Kim SH, Kim YK, et al. Imaging features of small $(\leq 3 \mathrm{~cm})$ pancreatic solid tumors on gadoxetic-acid-enhanced MR imaging and diffusion-weighted imaging: an initial experience. Magn Reson Imaging 2012; 30: 916-925.

12. Humphrey PE, Alessandrino F, Bellizzi AM, et al. Non-hyperfunctioning pancreatic endocrine tumors: multimodality imaging features with histopathological correlation. Abdom Imaging 2015; 40: 2398-2410.
13. Jeon SK, Lee JM, Joo I, et al. Nonhypervascular pancreatic neuroendocrine tumors: differential diagnosis from pancreatic ductal adenocarcinomas at MR imaging-retrospective cross-sectional study. Radiology 2017; 284: 77-87.

14. Ducreux M, Cuhna AS, Caramella C, et al.; ESMO Guidelines Committee. Cancer of the pancreas: ESMO Clinical Practice Guidelines for diagnosis, treatment and follow-up. Ann Oncol 2015; 26 (Suppl 5): v56-v68.

15. Hattori Y, Gabata T, Matsui O, et al. Enhancement patterns of pancreatic adenocarcinoma on conventional dynamic multi-detector row CT: correlation with angiogenesis and fibrosis. World J Gastroenterol 2009; 15: 3114-3121

16. Erkan M, Hausmann S, Michalski CW, et al. How fibrosis influences imaging and surgical decisions in pancreatic cancer. Front Physiol 2012; 3: 389.

17. d'Assignies G, Couvelard A, Bahrami S, et al. Pancreatic endocrine tumors: tumor blood flow assessed with perfusion CT reflects angiogenesis and correlates with prognostic factors. Radiology 2009; 2 : 407-416.

18. Yao XZ, Yun H, Zeng MS, et al. Evaluation of ADC measurements among solid pancreatic masses by respiratory-triggered diffusion-weighted MR imaging with Inversion-recovery fat-suppression technique at 3.0T. Magn Reson Imaging 2013; 31: 524-528.

19. Lotfalizadeh E, Ronot M, Wagner M, et al. Prediction of pancreatic neuroendocrine tumour grade with MR imaging features: added value of diffusion-weighted imaging. Eur Radiol 2016; 27: 1748-1759.

20. Wang Y, Chen ZE, Yaghmai V, et al. Diffusion-weighted MR imaging in pancreatic endocrine tumors correlated with histopathologic characteristics. J Magn Reson Imaging 2011; 33: 1071-1079.

21. Jang KM, Kim SH, Lee SJ, et al. The value of gadoxetic acid-enhanced and diffusion-weighted MRI for prediction of grading of pancreatic neuroendocrine tumors. Acta Radiol 2014; 55: 140-148. 